\title{
Contour Crafting Process Planning and Optimization
}

\author{
Jing Zhang and Behrokh Khoshnevis
}

Epstein Department of Industrial \& Systems Engineering, University of Southern California, Los Angeles, CA 90045 jingzhan@usc.edu,khoshnev@usc.edu

\begin{abstract}
Contour Crafting is an emerging technology that uses robotics to construct free form structures by repeatedly laying down layers of material such as concrete. The Contour Crafting technology scales up the additive fabrication process from building small industrial parts to constructing buildings. Tool path planning and optimization for Contour Crafting benefit the technology by increasing the efficiency of construction of complicated structures. This research has intended to provide a systematic solution for improving the overall system efficiency and realizing the automation of the Contour Crafting technology for building custom-designed houses. An approach is presented to find the optimal tool path for the single nozzle Contour Crafting system incorporating the physical constraints of the technology and construction considerations. Several algorithms are given to find the collision-free tool path for the multiple nozzle system based on the single nozzle approach.
\end{abstract}

\section{Introduction to Contour Crafting}

Contour Crafting [R2] can automatically construct custom-designed structures by repeatedly laying down construction material. It is an additive fabrication technology that uses computer control to exploit the superior surface-forming capability of troweling in order to create smooth and accurate planar and free form surfaces out of extruded materials. Unlike many other automatic additive fabrication technologies such as 3D printing, SLS, SLA, FDM[R4], which can only deliver relatively small size of three-dimensional structures (normally 1 cubic foot maximum), Contour Crafting has the capability to fabricate with thick layers using various materials and without compromising surface quality. Contour Crafting scales up the additive fabrication process to mega scale construction activities (Figure 1). The goal of Contour Crafting technology is to build custom-designed houses in a short time such as a day.

Since Contour Crafting has the ability of remarkably reducing the overall cost, injury, construction waste and impact to the environment, it can be effectively used for building houses for the low income class, shelters for disaster victims or even colonies on remote areas or other planets[R6]. Contour Crafting will also impact the construction industry for its capability and flexibility in constructing intricate or innovative structures. Its ability to build free-form shapes by utilizing the side trowels reduces the difficulty and cost of construction of complex structures. The cost of a house built by Contour Crafting technology mainly depends on the materials used and on the overall machine time. Innovative or organic form structures (such as adobe) might cost the same or even less than conventional rectangular structures because they require less support material. Architects are given more design flexibility because Contour Crafting eliminates many design limitations. CC allows architects to focus on the aesthetic appearance and functionality of the structure with less concern about construction limitations.

\section{Process planning and optimization in Contour Crafting}

Process planning and optimization play important roles in realizing the automation of the Contour Crafting system and improving the overall system efficiency. These functions generate optimal tool path for Contour Crafting system specific to the given structure designs. Furthermore, multiple-nozzle or multiplegantry systems may be involved in construction of larger community and multi-residence structures. In these cases specific schedule and workload will be assigned to individual nozzles or gantries for collaborative operation. Collision between nozzles should be avoided without compromising the overall constructing efficiency. This paper intends to present a systematic methodology for Contour Crafting process planning and optimization through the following steps: 
1. Describe system characteristics and define tool path elements of Contour Crafting

2. Develop practical tool path planning and an optimization method for the single nozzle CC system

3. Develop practical tool path planning and optimization methods for multi-nozzle system based on the optimization method for single nozzle

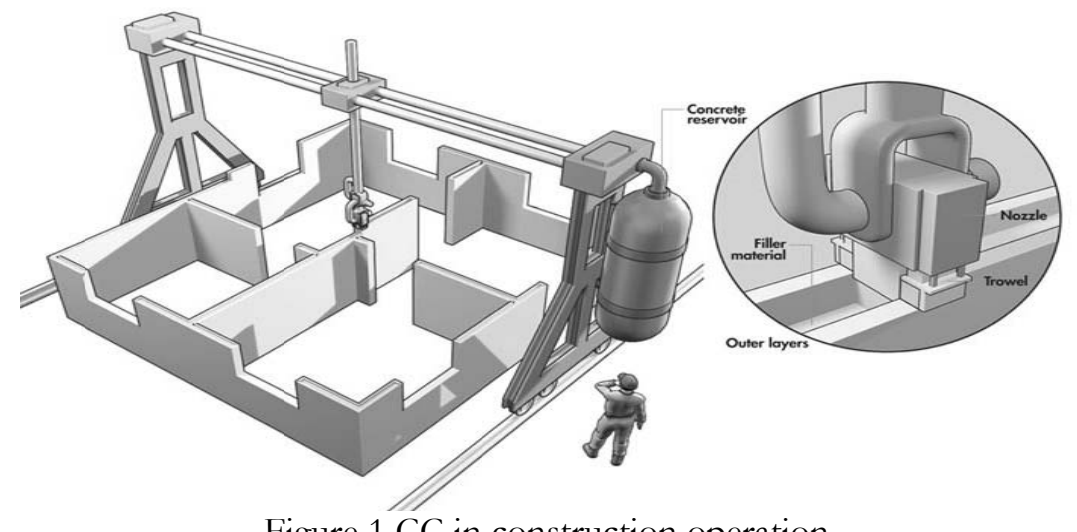

Figure $1 \mathrm{CC}$ in construction operation

\section{Implementation of process planning and optimization}

\subsection{System characteristics and tool path elements of Contour Crafting}

A Contour Crafting tool path for a specific structure must describe the position, orientation, velocity, and deposition rate of the nozzle in the entire construction period. This information is then converted to a sequence of machine tasks and then fed to the Contour Crafting machine. If we define the time or energy spent on each machine task as cost in general, then the goal of optimization would be finding a path with minimum total cost associated with every machine task. Therefore, cost of deposition, airtime and other machine tasks need to be defined for calculating the overall cost for the tool path.

Cost of deposition is related to the total length of wall segments, the deposition flow rate and the moving speed of the machine. Cost of airtime is related to the cost of moving between wall segments and the cost of rotation along wall segments. Cost of moving between two segments can be determined once the distance between end points and the velocity of the machine are known. Cost of rotation between segments can be evaluated according to the relative orientation of the two segments. However, in the real system, the degree of rotation of the nozzle is limited because the hoses and wires attached to the nozzle may tangle and become damaged if the nozzle rotates without any limitation. For this reason a mechanical stop is used on the rotation union to prevent the nozzle from turning more than 360 degrees in either direction. Nozzle rotation direction and degree of rotation need to be adjusted if the mechanical stop impedes the re-orientation transition of the nozzle in a given direction. Therefore, cost of rotation depends on not only the rotation degree but also on the start and end positions of the stopper on the rotation union. Cost of rotation between each pair of wall segments needs to be calculated before optimization is performed.

\subsection{Tool path planning and optimization method for the single nozzle CC system}

Once the costs of different machine tasks and physical constraints have been defined, optimization can be performed to find the most efficient tool path for the single nozzle system. The approach presented here is to convert the CC path model to a standard TSP (traveling salesman problem). This approach considers all the possible alternatives of construction and provides optimal solution if the TSP model is solved exactly. Heuristic TSP solvers can be used if the scale of the problem is large (for instance, the structure layout has more than 1000 vertices).

TSP is used to find the shortest route to visit a collection of cities at least once and return to the starting city. In the standard TSP problem, vertices represent cities while arcs are the paths between cities. In a 
standard TSP, distances between two cities are the same in both directions; otherwise we have an asymmetric TSP. A solution to the TSP must return the cheapest Hamiltonian cycle of the graph which represents the cities and paths. A Hamiltonian cycle is a simple path in the graph that contains each vertex. An asymmetric TSP problem can be formulated as follows: Define Xij $=1$ (when i,j are the index of the vertices), if edge (i,j) is in the optimal tour; otherwise $\mathrm{Xij}=0$, and $\mathrm{Dij}=\mathrm{d}(\mathrm{i}, \mathrm{j})$, when $\mathrm{d}$ is the traveling cost between vertices $\mathrm{i}$ and j. we have

$\operatorname{Min} \Sigma \Sigma \mathrm{Dij} \mathrm{Xij}$

$$
\begin{array}{ll}
\Sigma X_{\mathrm{ij}}=1 & \text { for all } \mathrm{j} \\
\Sigma \mathrm{Xij}=1 & \text { for all } \mathrm{I} \\
\Sigma \Sigma \mathrm{Xij}>=1 & \text { for every } \mathrm{S} \subseteq \mathrm{X} \text { (when } \mathrm{i} \in \mathrm{S} ; \mathrm{j} \in \mathrm{X} \text {-S) }
\end{array}
$$

The graph of a structure layout cannot be directly formulated as a standard TSP problem. In the CC construction process, some edges in the graph have to be traversed by the nozzle in order to deposit concrete for building walls, which means that the CC tool path has to contain some specific edges. However, any edge can be included in the optimal path in TSP since any edge represents a path between two cities. Also, a vertex in a structure layout may have several edges incident to it, which means during the construction process, the nozzle of the CC machine will visit the same vertex more than once. However, in TSP, each vertex can be visited only once. Figure 2 shows two graphs that share the same set of vertices. One of the graphs is a structure layout for CC. Another one is the optimal TSP path generated by Concorde TSP solver [R5], using the same set of vertices.

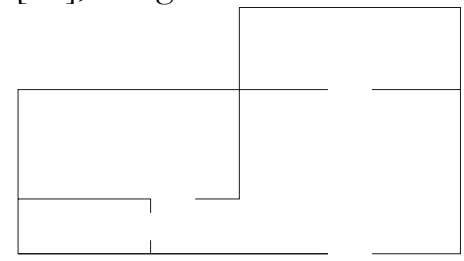

Layout model for CC construction

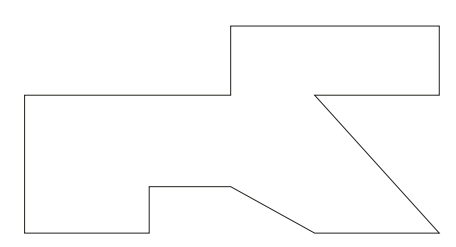

Tool path generated by Concorde TSP solver

Figure2: two graphs that share the same set of vertices construction

For Contour Crafting, the overall construction time of a specific structure is the sum of the overall time of concrete deposition and the overall nozzle airtime, in which the nozzle stops depositing material and travels between two deposition edges. No matter how the optimal path is generated, the nozzle should traverse all the deposition edges once and only once. The overall deposition time is determined once the structure is given. The overall nozzle idle time is the factor that determines the overall construction time for different tool paths. The optimal tool path is a path that has the minimum overall nozzle airtime. Since the nozzle of the machine can move freely in 3-dimensions, it can go straight between any vertices. The problem of finding the optimal tool path can be stated as follows:

Given a set of edges on a layout, find the optimum sequence and direction in which: (1) each edge is traversed exactly once and (2) the airtime travel (motion between two end points of two edges) is a straight line. The optimal solution minimizes the overall airtime travel.

An approach to formulate the problem is to ignore the deposition edges (walls) while only considering the traveling paths between edges (the airtime of the nozzle). In this case, walls shrink to vertices (entities), when the paths between vertices represent the cost of traveling between walls. Figure 3 shows the concept behind this approach.

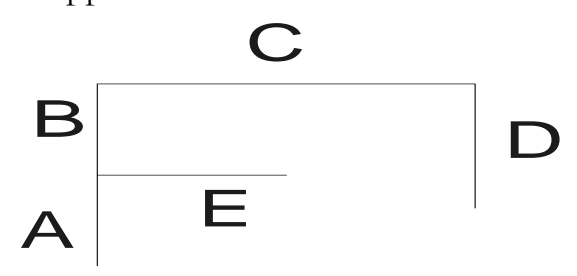

A structure layout

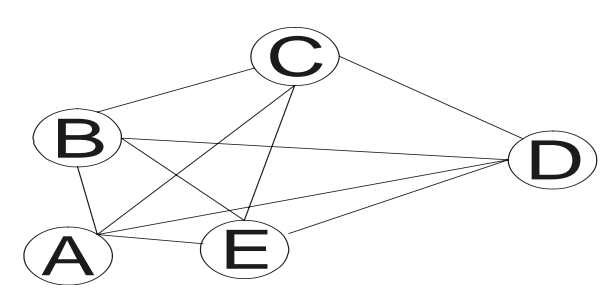

Compact edge to vertex (letters represent edges)

Figure 3 Concept of shrinking edges 
Since each edge has two vertices, the approach of shrinking the edge to a single point will have four possibilities to travel from one edge to another. As defined in Section 3.1, the cost of traveling from one wall segment to another depends on the time spent on moving and rotating the nozzle. Cost of rotation depends on the orientation of the two edges, the traveling sequence and the starting position of the stopper on the rotation union. Cost of rotation in opposite directions may be different even with the same rotation degree. Therefore, cost of rotation cannot be determined before performing the optimization. In order to formulate the problem as a TSP, some modifications need to be done:

Let Vi1 and Vi2 denote the two end points of the ith edge $(i=1 ; 2 ; \ldots ; n)$. Let $C(x, y)$ denote the traveling cost between points $\mathrm{x}$ and $\mathrm{y}$, which is determined by the rotation cost and the Euclidean distance of point $x$ and $y$. Define a complete network with vertex set $\{$ Vik $\mid i=1,2, \ldots, n ; k=1,2\}$. Between every pair of distinct vertices $(\mathrm{Vik}, \mathrm{Vjl})$ there is an undirected edge with length given by:

$\mathrm{C}(\mathrm{Vik}, \mathrm{Vjl})=-\mathrm{M}$ $=$ Traveling cost of Vik and Vij

$$
\text { if } i \neq j \text { if } i=j
$$

Where $\mathrm{M}$ is a large number (for example, $\mathrm{M}$ may be set equal to the total length of any feasible tour in the original problem). For $\mathrm{i}=1 ; 2 ; \ldots ; \mathrm{n}$, the distance of $-\mathrm{M}$ between vertices Vi1 and Vi2 implies that the optimal tour must include the edge that connects them. Therefore, every deposition edge will be traversed by the nozzle. A minimum length Hamiltonian cycle in this network yields a practical optimal tour for the tool path optimization problem. Figure 4 and Figure 5 show the concept behind this approach

Traveling Cost between

end points of an edge equals to $-\mathrm{M}$
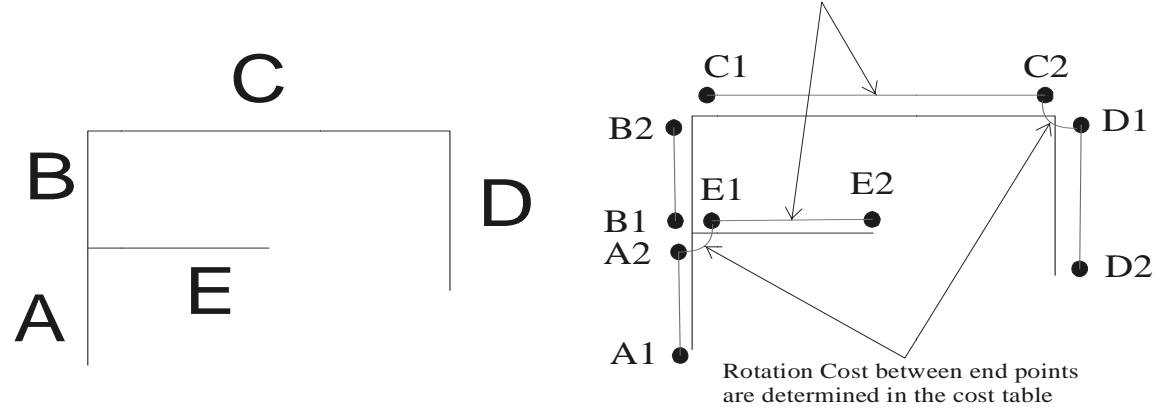

Figure 4 the concept of converting building layout to standard TSP problem.

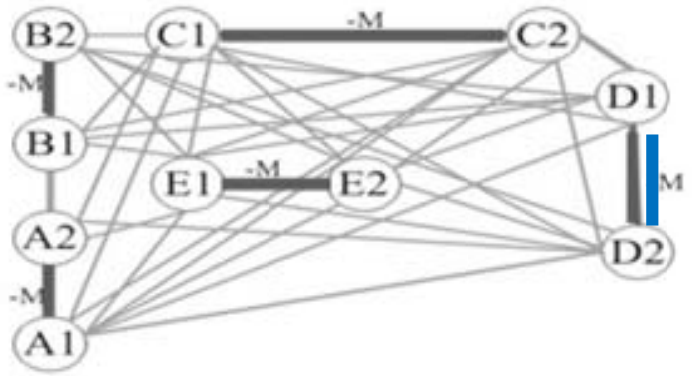

Figure 5 Traveling cost between end points of edges (blue/bold lines) equals to $-\mathrm{M}$, Other traveling costs (green/thin lines) are defined in Section 3.1.

The converted CC-TSP problem can be solved by using heuristic algorithms. Most TSP solvers use effective heuristic algorithms to find the acceptable result (normally no more than $5 \%$ of the optimal solution [R3]) within reasonable time. The Lin-Kernighan algorithm [R3] has been one of the most successful tour-improving methods during the 1970's and the 1980's. The two most recent implementations of Lin-Kernighan algorithm are the Chained (sometimes also called Iterated) Lin-Kernighan algorithm by Johnson and McGeoch [R1] and the modified Lin-Kernighan algorithm introduced by Helsgaun [R1]. The former changes the classic Lin-Kernighan algorithm by having it iterating in several steps. Helsgaun make some improvements on the original Lin-Kernighan algorithm, mainly by revising restrictions and directing the search for tour parts probably belonging to the optimal solution. Helsgaun's application is used in this research. 
Results: 50 structure layouts (small scale problem, less than 100 vertices) have been tested using the above approach with the given CC system parameters. Single nozzle optimal tool paths have been successfully found for all the layouts. CPLEX [R5], a commercial integer programming solver is used to check the accuracy of the result.

\subsection{Tool path planning and optimization methods for multi-nozzle system based on optimization of the single nozzle case}

The primary concern in using multiple nozzles (or gantries) is that collision between different nozzles/gantries should be avoided. The tool path generation of the multi-nozzle system includes two steps. The first step is to separate the original structure into different sections according to the number of nozzles by using an iterative dividing procedure. The second step is to create tool paths for these sections so that no collision between the nozzles occurs when they travel along the tool paths.

\subsubsection{Step1: Iterative dividing.}

In order to assign workloads to different nozzles, the original structure layout should be separated into different sections according to the number of the nozzles. Ideally, each section contains an equal amount of work load so that the construction time of all of the sections is the same. Straight lines can cut across the original layout in order to divide it into sections with the condition that the sums of the length of all of the wall segments in different sections are equal or approximate. The single nozzle optimization algorithm (CCTSP) is applied to find out the overall construction time of each section of the layout. If the difference between the construction times is acceptable (lower than the pre-set threshold) then the workload assignment is considered to be achieved. Otherwise, the cutting lines should be moved and split the original structure, the optimization should be performed again on each section to find the difference between the construction times. The above procedures will be performed iteratively until the best result is achieved.
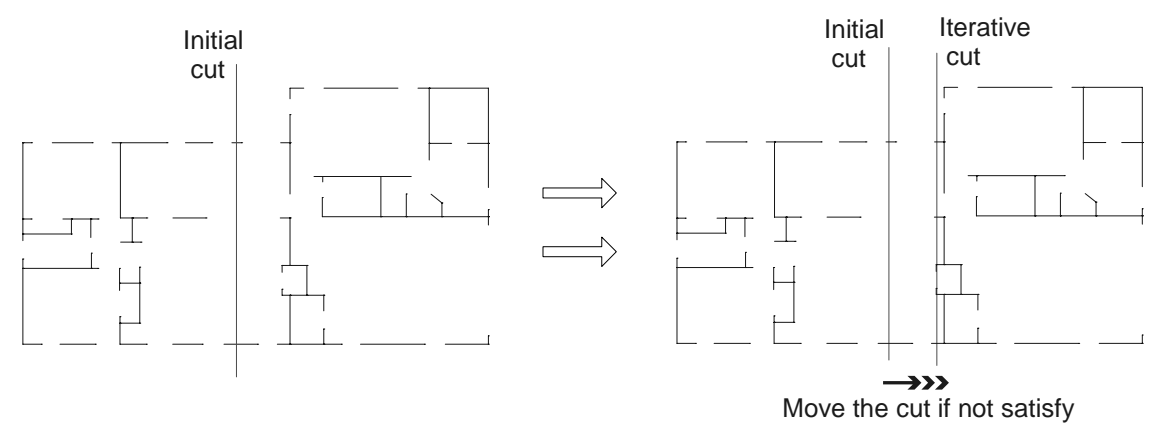

Figure 6 Iterative dividing

\subsubsection{Step 2: create collision-free tool paths between the divided parts}

After evenly dividing the structure into different sections, collision-free tool paths between the divided sections can be created. There are two ways to prevent collisions during the construction, they are: (1) setup a buffer area to prevent the nozzles from getting too close to each other during the construction process, and (2) analyze the $\mathrm{x} / \mathrm{t}$ curves of the gantries that carry the nozzles. Three algorithms are proposed to find the optimal collision-free tool paths. Some algorithms have a higher chance of converging to a feasible solution than the others. However, the extent of optimality of their solutions might be lower. These algorithms are: (1) buffer zone; (2) path cycling; (3) buffer zone path cycling.

\subsubsection{Buffer zone}

Nozzles may collide near the shared section borders. Gantries that carry the nozzles could collide with each other when they are working near the cutting edge of adjacent sections since the width of the gantries is not equal to zero. See figure 7.

Buffer zones can be setup on both sides of the shared border in order to prevent collisions near the border. Buffer zones must meet the following conditions: (1) the size (width) of the buffer zone should be bigger than the width of the gantry; (2) the overall workload in the buffer zone should be less than half of the overall workload within the section that contains the buffer zone. When more than two gantries are working together, one gantry should avoid the collision with gantries on either side, therefore each divided section needs to have two buffer zones. The concept of auxiliary buffer zone can be used to reduce the 
number of buffer zones (See figure 8). First a buffer zone is generated for each section, and then the construction time of each buffer zone will be calculated. If the construction time of a buffer zone of a section is bigger than that of a buffer zone of the next section, no additional buffer zone is needed for that section. Otherwise, auxiliary buffer zones should be generated next to buffer zone with less construction time. In each section, the nozzle should work according to the following order: (1) buffer zone, (2) auxiliary buffer zone (if there is any) and (3) the main working zone. These constraints assure that the working areas of any two nozzles are mutually exclusive during the entire operation; therefore, collisions can be avoided.
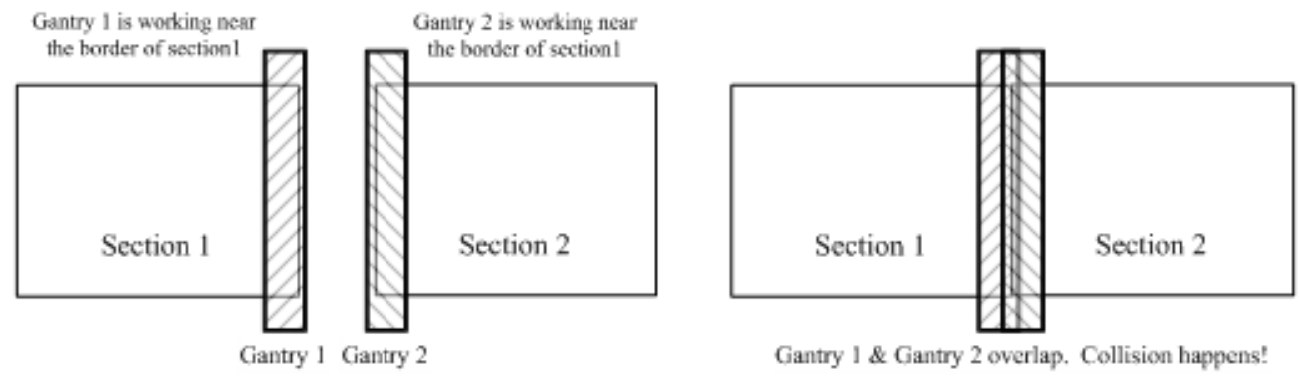

Figure 7 Possible collisions between two gantries

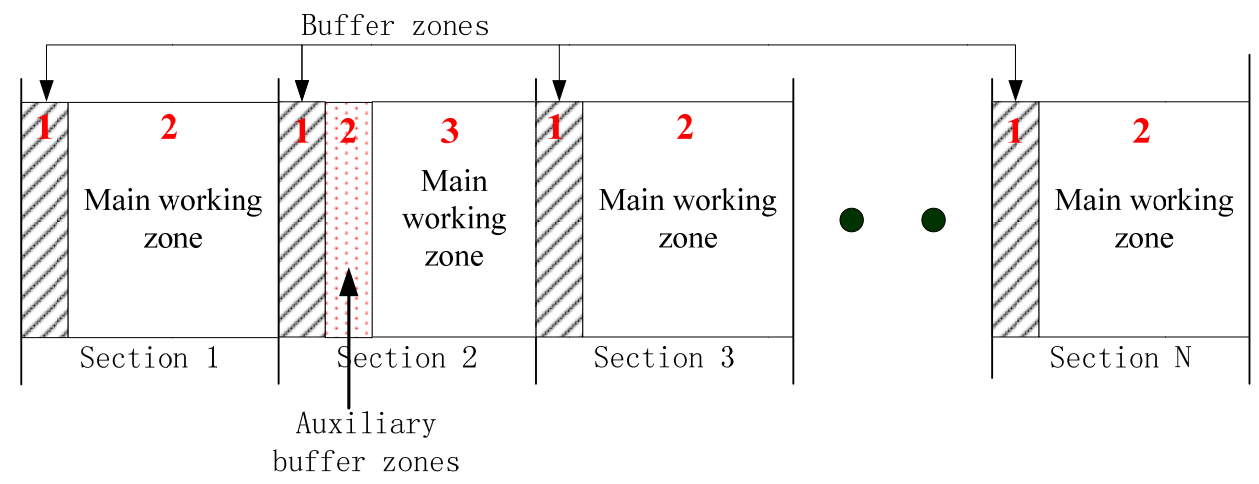

Figure 8 Auxiliary buffer zone. (numbers are the construction sequence of a nozzle)

\subsubsection{Path cycling}

Since the nozzles are carried by their corresponding gantries, two nozzles never collide if the distance between the corresponding gantries is never smaller than a specific amount in anytime. Let $x 1(t), x 2(t)$ represent the $x$ position of the nozzle 1 and nozzle 2 in time t, we have:

$\mathrm{x} 1(\mathrm{t})-\mathrm{x} 2(\mathrm{t})<$ Specific Distance (to prevent the collision), When $0<\mathrm{t}<$ end of the construction

An $x / t$ curve can represent the $x$ position of a nozzle at time $t$. If two $x / t$ curves never cross each other and the minimal distance between these curves is never smaller than a specific amount, then the two nozzles will not collide with each other during the entire construction process. If these two curves can be constructed and the overall time of the longer curve is minimized, then the optimal solution will be yielded. (See figure 9)

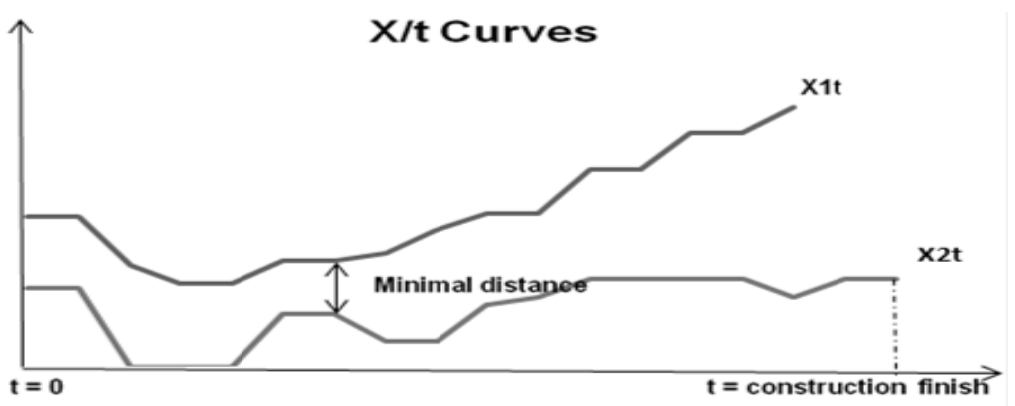

Figure $9 \mathrm{x} / \mathrm{t}$ curves 
Since the optimal CC-TSP tool path is a loop (the nozzle will visit the starting point at the end), cycling the tool path will not increase the overall construction time. We can therefore always cycle one of the paths to increase the chance of finding a pair or collision-free $\mathrm{x} / \mathrm{t}$ curves. To cycle a path, the first vertex is placed at the end of the sequence, but the sequence of the vertices remains the same in the tool path. The next consecutive vertex is then moved to the end of sequence if the cycling is continuous. The two paths will be checked during the cycling to see if they will collide. The cycling process will be complete if the sequence returns to its original pattern.

There are two ways to manipulate the $\mathrm{x} / \mathrm{t}$ curve of the tool path to avoid collision. Global path cycling is achieving the result directly from the CC-TSP tool path for the single nozzle system. The optimal tool path for the entire structure is split into different tool paths according to the number of nozzles. Path cycling will be performed to find the collision-free tool path pairs between the split paths. Individual path cycling is similar to global path cycling. Instead of separating the global optimal tool path, wall segments of the original structure layout are sorted and assigned into different groups. The optimization is then performed on each of the two groups to find the local CC-TSP tool paths. These tool paths are then checked to detect if they cross each other. If they do, one of the paths is cycled and checked again until either the solution is found or the cycling process is complete (i.e., failure in finding a solution). This algorithm has a higher chance to find the result, yet the total construction time of the final solution may be longer than that of global path cycling. Figure 10 shows the concept of individual path ycling.

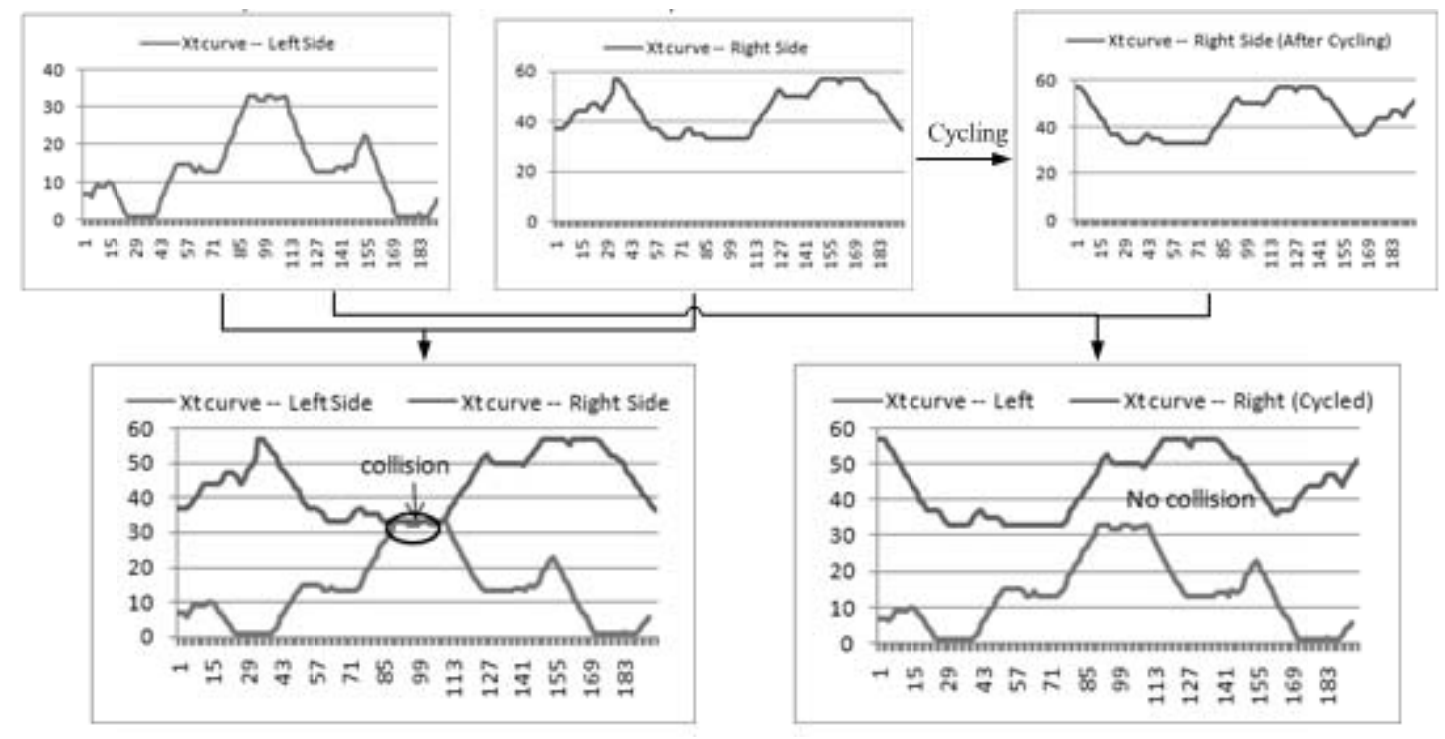

Figure 10 Individual path cycling

\subsubsection{Buffer zone path cycling}

The method of path cycling can create collision-free tool paths from the CC-TSP paths in most of the cases. However, the chance of finding the collision-free tool paths still depends on the geometry of the structure and the width of gantry to a certain degree. There is a higher chance to find a solution by combining the concept of the buffer zone and path cycling.

When multiple machines are used in construction, the structure is first evenly divided into sections according to the number of machines. Each section will be cut or separated into two zones: the buffer zone and the main working zone. CC-TSP tool paths will be generated for both zones of each section, and then the tool path of the working zone will be cycled to avoid collision with the buffer zone next to it. Unlike the previous method (path cycling), the procedure for making sure that the adjacent paths do not collide are independent from each other in the method of buffer zone path cycling. Only the paired up working zone path and buffer zone path will be checked. The purpose of cycling the path of the working zone is only to increase the chance to create collision-free tool paths with the buffer zone with which it is paired up and will not cause any collisions with any other tool paths. This will dramatically increase the chance of finding collision-free tool paths when many machines are involved in construction.

3.3.3 Results: 
50 random structure layouts each having less than 200 vertices have been tested using the above algorithms with the given CC system parameters. Following are the number of collision-free tool paths for each algorithm listed:

23 for buffer zone, 12 for global path cycling, 42 for Individual path cycling and 50 for buffer zone path cycling (with the longer overall airtime than cycling based global optimization).

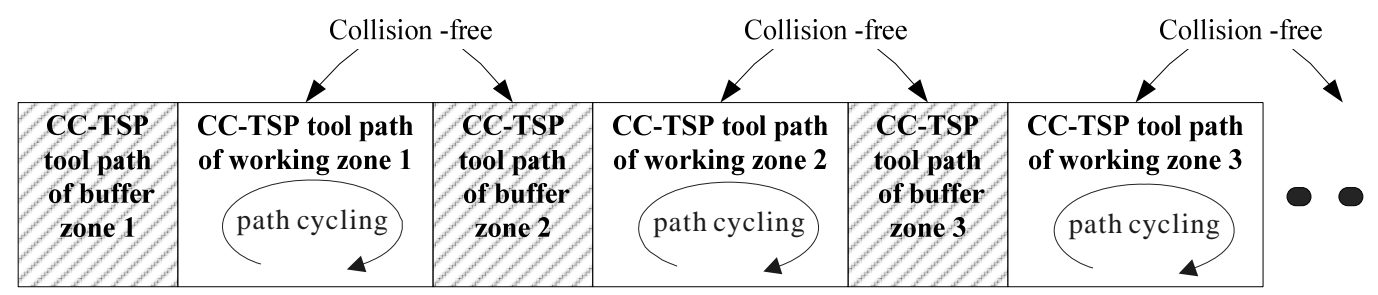

Figure 11 Buffer Zone Path Cycling for $\mathrm{N}$ machines system

\section{Conclusion}

This research has intended to provide a systematic solution for improving the overall efficiency of construction by Contour Crafting. An approach is presented to find the optimal tool path for the single nozzle Contour Crafting system. Several algorithms are also presented to find the collision-free tool path for the case of multiple nozzle systems. Practical and efficient tool paths can be generated using the proposed approaches to enhance the already attractive aspects of Contour Crafting.

\section{Reference}

[1] K. Helsgaun, "An effective implementation of the Lin-Kernighan traveling salesman heuristic", European Journal of Operational Research. 126, no. 1, October 2000, pp. 106-130.

[2] B. Khoshnevis, (2004) 'Automated construction by contour crafting-related robotics and information technologies' Automation in Construction, Vol. 12, pp. 5-19

[3] S. Lin, S. and B. Kernighan, "An Effective Heuristic Algorithm for the Traveling Salesman Problem", Operations Research, vol. 21, 1973, pp. 498-516.

[4] Pease III, L.F., "Rapid Prototyping Methods," ASM Handbook Volume 7:

[5] Powder Metal Technologies and Applications, 1998.

[6] Concorde TSP solver. http://www.tsp.gatech.edu/concorde.html

[7] Z. Yeh, "Trowel-Path Planning For Contour Crafting", Ph.D. Dissertation, University of Southern California, 2003 